\section{Single nucleotide polymorphism discrimination assisted by improved base stacking hybridiza- tion using oligonucleotide microarrays}

\author{
D. Wang ${ }^{1,2}$, H. Gao ${ }^{1,2}$, R. Zhang2, X. Ma², Y. Zhou ${ }^{1,2}$, and J. Cheng ${ }^{1,2}$ \\ ${ }^{1}$ Tsinghua University and ${ }^{2}$ National Engineering Research Center for Beijing Biochip \\ Technology, Beijing, P.R. China
}

BioTechniques 35:300-308 (August 2003)

Efficiencies of mismatch discrimination using size-varied capture probes were examined at various hybridization temperatures. The probes were 17, 15, 13, 11, 9, and 7 nucleotides long and contained single-base mismatches at their 3'ends. The optimal signal intensity and efficiency of base stacking hybridization on mismatch discrimination were observed for capture probes with a melting temperature $\left(T_{m}\right)$ value of $36^{\circ} \mathrm{C}$, in the detection of DNA sequence variations at $40^{\circ} \mathrm{C}$. We employed asymmetric PCR to prepare single-stranded target DNA labeled with a fluorescent dye, and the PCR product was hybridized on the DNA microarray with no further purification. Our efforts have enhanced the sensitivity and simplified the procedures of base stacking hybridization on mismatch discrimination. As a model experiment, this improved technology was used to identify plasmid templates of human leukocyte antigen (HLA)A alleles 2601, 2902, and 0206 on oligonucleotide microarrays. It is now possible to apply this simple, rapid, sensitive, and reliable base stacking hybridization technology to detect DNA sequence variations on microarrays in clinical diagnosis and other applications.

\section{INTRODUCTION}

Stacking interactions between adjacent bases play an important role in the formation of nucleic acid duplexes (1). Several studies of stacking interactions in RNA and DNA have been reported to date (2-5). The term base stacking hybridization was coined to describe the additional stability of DNA achieved by hybridization between two or more contiguously tandem DNA oligonucleotides and a larger complementary single-stranded DNA (ssDNA) (6).

Base stacking hybridization has been used to increase the efficiency of discrimination between perfect and mismatched DNA duplexes on 3-D gel pad microchips (1,6-13). Recently, capture probes containing polymorphic sites immobilized on microarrays were employed to hybridize with target DNAs to detect mutations assisted by stacking oligonucleotides $(14,15)$. This method requires only one round of hybridization and is simpler than all the previous protocols $(14,15)$. The results of mismatch discrimination studies with base stacking hybridization on mi- croarrays were affected by several factors: $(i)$ the difference in the adjacent bases between the capture probe and stacking oligonucleotide; (ii) the types of base mismatches; and (iii) the length of the capture probes. Although the first two factors have been intensively studied, few papers have dealt with the last factor. Maldonado-Rofriguez and coworkers $(14,15)$ have investigated changes of mismatch discrimination using microarrays that contained capture probes of different lengths (5-9 nucleotides), and their results suggested that capture probes with 9 nucleotides were the better ones. However, short capture probes of fewer than 10 nucleotides have low hybridization sensitivity and efficiency (16). In addition, traditional base stacking hybridization procedures used to identify sequence variations on microarrays were tedious and time-consuming, especially in the preparation of singlestranded target DNAs.

The goal of our study was to examine the efficiency and sensitivity of mismatch discrimination using base stacking hybridization on microarrays with size-varied capture probes. We sought to develop a simplified protocol to make this method more efficient, sensitive, and reliable when combined with the DNA microarray technology that could be employed for clinical diagnosis and other applications in the future.

\section{MATERIALS AND METHODS}

\section{Oligonucleotides}

All oligonucleotides were synthesized, labeled, and gel-purified by BioAsia (Shanghai, China). Table 1 lists the names, sequences, and intended roles of these oligonucleotides.

\section{Fluorescence-Labeled Asymmetric PCR}

Plasmids containing three human leukocyte antigen (HLA)-A genotypes (0206, 2601, and 2902) were used as templates. PCR amplifications were carried out in a $50-\mu \mathrm{L}$ total reaction volume containing $1 \times$ PCR buffer (Takara, Dalian, China), $0.2 \mathrm{mM}$ dNTPs, $0.02 \mathrm{mM} \mathrm{Cy}^{\mathrm{TM}} 5$-dCTP, $0.5 \mathrm{U}$ Taq DNA polymerase (Takara), $0.5 \mu \mathrm{M}$ HLA-primer-1, and $0.02 \mu \mathrm{M}$ HLAprimer-2 on a Perkin Elmer 9600 thermal cycler (Perkin Elmer Life Sciences, Gaithersburg, MD, USA). PCR temperature and time sequences were $96^{\circ} \mathrm{C}$ for $3 \mathrm{~min}$, followed by 25 cycles of $96^{\circ} \mathrm{C}$ for $25 \mathrm{~s}, 71^{\circ} \mathrm{C}$ for $45 \mathrm{~s}, 72^{\circ} \mathrm{C}$ for $30 \mathrm{~s}$, and then 8 cycles of $96^{\circ} \mathrm{C}$ for $25 \mathrm{~s}, 68^{\circ} \mathrm{C}$ for $1 \mathrm{~min}, 72^{\circ} \mathrm{C}$ for $2 \mathrm{~min}$, with a final extension step at $72^{\circ} \mathrm{C}$ for $10 \mathrm{~min}$.

\section{Construction of Oligonucleotide Microarrays}

Capture probes were dissolved in $50 \%$ dimethyl sulfoxide (DMSO) to make $10-\mu \mathrm{M}$ working solutions. A commercial arrayer, the PixSys5500 (Cartesian Technologies, Irvine, CA, USA), was employed to spot oligonucleotides onto aldehyde-modified glass slides. The spot size was approximately 150 $\mu \mathrm{m}$, and the center-to-center distance between adjacent spots was $300 \mu \mathrm{m}$. To verify the reproducibility, four spots were printed for each oligonucleotide on the same glass slide. 
Table 1. Names, Sequences, $T_{m}$ Values, and Intended Roles of Oligonucleotides

\begin{tabular}{|c|c|c|c|}
\hline Name & Sequence & $\mathrm{T}_{\mathrm{m}}\left({ }^{\circ} \mathrm{C}\right)$ & Role \\
\hline HLA-primer-1 & 5'-GGCCTCCCCAGCCGCCGAGGATGGCC-3' & - & PCR primer \\
\hline HLA-primer-2 & 5'-CGGGTCCCGTGGCCCCTGGTACCCG-3' & - & PCR primer \\
\hline HLASO1 & 5'-GAACCTGGGGACCCTGCGCGGCTACTAC-3' & - & Stacking oligonucleotide \\
\hline HLASO2 & 5'-TGTGGCGGAGCAGTGGAGAGCC-3' & - & Stacking oligonucleotide \\
\hline HLASO3 & 5'-GGACGGGCGCTTCCTCCGC-3' & - & Stacking oligonucleotide \\
\hline HLACP1 & $5^{\prime}-\mathrm{NH}_{2}-\mathrm{T}_{12}-\mathrm{TCACAGACTGACCGAGC-3^{ \prime }}$ & 54 & Capture probe \\
\hline HLACP2 & $5^{\prime}-\mathrm{NH}_{2}-\mathrm{T}_{12}-\mathrm{TCACAGACTGACCGAGG-3^{ \prime }}$ & 54 & Capture probe \\
\hline HLACP3 & $5^{\prime}-\mathrm{NH}_{2}-\mathrm{T}_{12}-\mathrm{TCACAGACTGACCGAGT-3^{ \prime }}$ & 52 & Capture probe \\
\hline HLACP4 & $5^{\prime}-\mathrm{NH}_{2}-\mathrm{T}_{12}$-ACAGACTGACCGAGC-3' & 48 & Capture probe \\
\hline HLACP5 & $5^{\prime}-\mathrm{NH}_{2}-\mathrm{T}_{12}$-ACAGACTGACCGAGG-3' & 48 & Capture probe \\
\hline HLACP6 & $5^{\prime}-\mathrm{NH}_{2}-\mathrm{T}_{12}-\mathrm{ACAGACTGACCGAGT-3^{ \prime }}$ & 46 & Capture probe \\
\hline HLACP7 & $5^{\prime}-\mathrm{NH}_{2}-\mathrm{T}_{12}-\mathrm{AGACTGACCGAGC-3^{ \prime }}$ & 42 & Capture probe \\
\hline HLACP8 & $5^{\prime}-\mathrm{NH}_{2}-\mathrm{T}_{12}-\mathrm{AGACTGACCGAGG-3^{ \prime }}$ & 42 & Capture probe \\
\hline HLACP9 & $5^{\prime}-\mathrm{NH}_{2}-\mathrm{T}_{12}$-AGACTGACCGAGT-3' & 40 & Capture probe \\
\hline HLACP10 & $5^{\prime}-\mathrm{NH}_{2}-\mathrm{T}_{12}-\mathrm{ACTGACCGAGC}-3^{\prime}$ & 36 & Capture probe \\
\hline HLACP11 & $5^{\prime}-\mathrm{NH}_{2}-\mathrm{T}_{12}-\mathrm{ACTGACCGAGG}-3^{\prime}$ & 36 & Capture probe \\
\hline HLACP12 & $5^{\prime}-\mathrm{NH}_{2}-\mathrm{T}_{12}-\mathrm{ACTGACCGAGT-3^{ \prime }}$ & 34 & Capture probe \\
\hline HLACP13 & $5^{\prime}-\mathrm{NH}_{2}-\mathrm{T}_{12}-\mathrm{TGACCGAGC-3^{ \prime }}$ & 30 & Capture probe \\
\hline HLACP14 & $5^{\prime}-\mathrm{NH}_{2}-\mathrm{T}_{12}-\mathrm{TGACCGAGG}^{\prime} 3^{\prime}$ & 30 & Capture probe \\
\hline HLACP15 & $5^{\prime}-\mathrm{NH}_{2}-\mathrm{T}_{12}-\mathrm{TGACCGAGT}^{\prime}$ & 28 & Capture probe \\
\hline HLACP16 & $5^{\prime}-\mathrm{NH}_{2}-\mathrm{T}_{12}-\mathrm{ACCGAGC}-3^{\prime}$ & 24 & Capture probe \\
\hline HLACP17 & $5^{\prime}-\mathrm{NH}_{2}-\mathrm{T}_{12}-\mathrm{ACCGAGG}-3^{\prime}$ & 24 & Capture probe \\
\hline HLACP18 & $5^{\prime}-\mathrm{NH}_{2}-\mathrm{T}_{12}-\mathrm{ACCGAGT-3^{ \prime }}$ & 22 & Capture probe \\
\hline HLACP19 & $5^{\prime}-\mathrm{NH}_{2}-\mathrm{T}_{12}$-AGGCGGCCCG-3' & 38 & Capture probe \\
\hline HLACP20 & $5^{\prime}-\mathrm{NH}_{2}-\mathrm{T}_{12}-\mathrm{AGGCGGCCCA-3^{ \prime }}$ & 36 & Capture probe \\
\hline HLACP21 & $5^{\prime}-\mathrm{NH}_{2}-\mathrm{T}_{12}$-GACGTGGGGT-3' & 34 & Capture probe \\
\hline HLACP22 & $5^{\prime}-\mathrm{NH}_{2}-\mathrm{T}_{12}$-GACGTGGGGC-3' & 36 & Capture probe \\
\hline
\end{tabular}

\section{Pre-Annealing of Stacking} Oligonucleotide to Target DNA

Stacking oligonucleotides were preannealed to the unpurified asymmetric PCR product in a $8.1-\mu \mathrm{L}$ reaction mixture containing $6 \times$ standard saline citrate (SSC), $0.1 \%$ sodium dodecyl sulfate (SDS), 2.78 pmol stacking probe, and $1.5 \mu \mathrm{L}$ unpurified PCR products. The pre-annealing conditions were $98^{\circ} \mathrm{C}$ for $3 \mathrm{~min}, 55^{\circ} \mathrm{C}$ for $15 \mathrm{~min}$, and $4^{\circ} \mathrm{C}$ for $5 \mathrm{~min}$.

\section{Hybridization and Washing}

The pre-annealed reaction mixture was allowed to hybridize to the oligonucleotide microarrays at the indicated temperature for $2 \mathrm{~h}$. When the hybridization was complete, the microarrays were washed in $50 \mathrm{~mL}$ washing buffer containing $0.1 \%$ SDS and $2 \times$ $\mathrm{SSC}\left(0.3 \mathrm{M} \mathrm{NaCl}, 0.03 \mathrm{M} \mathrm{Na}_{3}\right.$-citrate, $\mathrm{pH}$ 7.0) for $15 \mathrm{~min}$, followed by a double-distilled water rinsing at room temperature for $2 \mathrm{~min}$. The glass slides were centrifuged to dryness in $50-\mathrm{mL}$ conical tubes at $120 \times g$ for $1 \mathrm{~min}$.

\section{Array Scanning and Data Analysis}

The arrays were scanned on a ScanArray ${ }^{\circledR} 4000$ scanner (GSI Lumonics Kanata, Kanata, ON, Canada) (laser power, $70 \%$ and photomultiplier tube,
$57 \%$ ), and the obtained images were analyzed with a GenePix ${ }^{\circledR}$ Pro 3.0 software (Axon Instruments, Union City, CA, USA). In this report, the relative fluorescence intensity of a capture probe was defined as the signal after subtraction of half of the background fluorescence. Each data set was obtained from four identical arrays in two slides.

\section{RESULTS AND DISCUSSION}

\section{Effects of Hybridization Temperature}

Of the eight possible single-base mismatches, $\mathrm{G} / \mathrm{G}$ is one of the easiest mismatch types to discriminate, while 
Table 2. The Highest Discrimination Ratio

\begin{tabular}{|lccccc|}
\hline \multirow{2}{*}{$\begin{array}{l}\text { Capture } \\
\text { Probe Length } \\
\text { (Nucleotides) }\end{array}$} & \multicolumn{2}{c}{ G/G Mismatch } & & \multicolumn{2}{c|}{ G/T Mismatch } \\
\cline { 2 - 3 } \cline { 5 - 6 } & HR & HT $\left({ }^{\circ} \mathbf{C}\right)$ & & HR & HT $\left({ }^{\circ} \mathbf{C}\right)$ \\
\hline 7 & $2.1 \pm 1.6$ & 25 & $3.1 \pm 0.6$ & 25 \\
9 & $41.2 \pm 14.3$ & 25 & & $50.3 \pm 15.3$ & 25 \\
11 & $90.1 \pm 6.2$ & 40 & & $45.8 \pm 3.1$ & 40 \\
13 & $21.9 \pm 3.1$ & 55 & & $13.8 \pm 1.8$ & 55 \\
15 & $16.9 \pm 2.7$ & 60 & & $13.1 \pm 0.9$ & 60 \\
17 & $5.4 \pm 1.0$ & 60 & & $4.9 \pm 0.3$ & 65 \\
HR, highest ratio; HT, hybridization temperature. & & \\
\hline
\end{tabular}

$\mathrm{G} / \mathrm{T}$ is the most difficult one (16). In our study, we chose G/G and G/T mismatches as models to examine the efficiency of discrimination with capture probes of different lengths using base stacking hybridization on microarrays. The target DNA for hybridization was HLA-A type 2601, and the array was constructed by capture probes derived from HLACP1 to HLACP18. These probes are adjacent to HLASO1 in the complementary single-stranded HLA-A 2601.

Figure 1 shows that the fluorescence intensities of hybridization initially increased with temperature, followed by signal decreases in perfectly matched capture probes from $15^{\circ}-75^{\circ} \mathrm{C}$. Mismatch discriminations can be deter- mined by the ratios of relative signal intensities of perfectly matched hybridizations to mismatched ones obtained under the same experimental conditions. Table 2 lists the temperatures to obtain the best discrimination on $\mathrm{G} / \mathrm{G}$ mismatch and $\mathrm{G} / \mathrm{T}$ mismatch with $17,15,13,11,9$, and 7 nucleotide capture probes.

\section{Effects of the Capture Probe Length}

For the G/G mismatch and G/T mismatch, the optimal signal ratios of perfectly matched and mismatched duplexes have been listed (Table 2). The highest discrimination efficiency was obtained for a capture probe of 11 nucleotides. The hybridization signal of

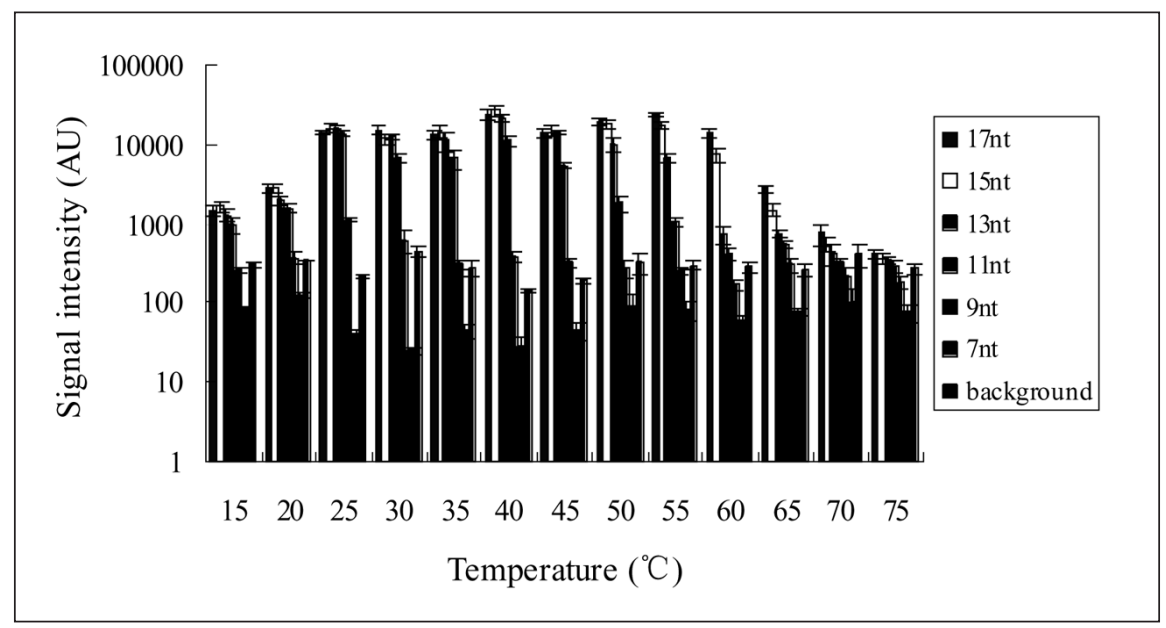

Figure 1. Effects of hybridization temperature and capture probe length on the signal intensity of perfectly matched capture probes on microarrays. The target DNA sequence (283-327) complementary to capture probes and stacking oligonucleotide HLASO1 was 5'-GTAGTAGCCGCGCAGGGTCCCCAGGTTCgCTCGGTCAGTCTGTGA-3', where g is a single-base variation in HLA-A 2601 DNA sequence. Capture probes immobilized on microarrays were derived from HLACP1 to HLACP18. Note that the scale of the ordinate is logarithmic. AU, arbitrary unit; nt, nucleotide.
9- and 7-nucleotide capture probes was so low that their discrimination ratios could not represent their true discrimination efficiency in this study (Table 3 ).

Our results indicated that the 11and 13-nucleotide capture probes, with single base mismatches at their $3^{\prime}$ terminals, could clearly discriminate perfectly matched duplexes from $\mathrm{G} / \mathrm{G}$ or $\mathrm{G} / \mathrm{T}$ mismatched duplexes. For the sake of higher ratio of signals from perfectly matched to mismatched duplexes, the 11-nucleotide capture probe is more suitable for base stacking hybridization studies than the 13-nucleotide capture probe.

The stability of DNA duplexes plays the most important roles in mismatch discrimination (3). The more stable the DNA duplexes are, the weaker the destabilizing effect of the mismatches. Usually, it is the melting temperature $\left(T_{m}\right)$ value and not the length of DNA duplexes that indicates the stability of DNA duplexes. Therefore, 11 nucleotides, the optimal length of the capture probe in this report, was not universal and will be changed in different conditions, such as different hybridization buffer and different sequences. The $\mathrm{T}_{\mathrm{m}}$ value of optimal capture probes may be more useful than the length of $11 \mathrm{nu}-$ cleotides. As listed in Table 1 , the $T_{m}$ values of perfectly matched capture probes of 11 and 13 nucleotides were $36^{\circ}$ and $42^{\circ} \mathrm{C}$, respectively. This provides a useful guide for designing capture probes in DNA base variation detection assisted by base stacking hybridization. Therefore, the length of the capture probes utilized in the HLA genotyping was 10 nucleotides because the $T_{m}$ value of these capture probes was approximately $36^{\circ} \mathrm{C}$.

\section{Single-Stranded Target DNA Preparation}

Unlike double-stranded DNA (dsDNA) targets, single-stranded target DNA can enhance hybridization efficiency remarkably on oligonucleotide microarrays. In previous reports of base stacking hybridization, single-stranded target DNA was mainly prepared by streptavidin-coated magnetic beads $(14,15,17,18)$ or by single-primer reamplifying PCRs, followed by purification using electrophoresis (7). Those 
Table 3. The Strongest Hybridization Signal with Different Capture Probes

\begin{tabular}{|lcccccc|}
\hline & \multicolumn{5}{c|}{ Capture Probe Length (Nucleotides) } \\
\cline { 2 - 7 } & $\mathbf{7}$ & $\mathbf{9}$ & $\mathbf{1 1}$ & $\mathbf{1 3}$ & $\mathbf{1 5}$ & $\mathbf{1 7}$ \\
\hline SHS (arbitrary units) & $123.4 \pm 10.4$ & $1078.7 \pm 63.6$ & $13442.0 \pm 1145.6$ & $21299.3 \pm 2474.5$ & $25876.1 \pm 3586.3$ & $23620.2 \pm 992.3$ \\
HT $\left({ }^{\circ} \mathrm{C}\right)$ & 20 & 25 & 25 & 40 & 40 & 55 \\
SHS, strongest hybridization signal; HT, hybridization temperature. & & & \\
\hline
\end{tabular}

methods were laborious and often yielded low amounts of ssDNA.

Here fluorescence-labeled asymmetric PCR was used to prepare ssDNA and to label the single-stranded target DNA with Cy5-dCTP simultaneously. In addition, the fluorescence-labeled asymmetric PCR product needed no further purification and could be hybridized directly to oligonucleotide microarrays after being pre-annealed with stacking oligonucleotides. Fluorescence-labeled asymmetric PCR significantly simplified the overall process of mutation and SNP detection using base stacking hybridization on microarrays, making it more suitable for disease diagnosis and other applications.

Based on previous reports, the labeling efficiency of target DNA in base stacking hybridization reactions was also low because one fluorescence molecule or one ${ }^{32} \mathrm{P}$ was usually labeled on one target molecule. Here the singlestranded target DNA was labeled with Cy5-dCTP through PCR, and one 1000nucleotide DNA molecule could be labeled with approximately $10 \mathrm{Cy} 5 \mathrm{~mol}-$ ecules (data not shown). In our study, the target DNA for hybridization was 1098 nucleotides; therefore, one target DNA molecule was labeled with approximately 10 Cy5 molecules. Using this improved method, only $1.5 \mu \mathrm{L}$ asymmetric PCR products were adequate for the hybridization against capture probes immobilized on planar microarrays.

\section{HLA Genotyping Using Base Stack- ing Hybridization on Microarrays}

HLA is one of the most important immune systems in the human body, and HLA genotyping plays an important role in the identification of suitably matched recipients for donors and patients $(19,20)$. The improved base stacking hybridization was applied to identi- fy HLA types of HLA-A 0206, 2601, and 2902 on microarrays. Figure 2 lists the detailed information of variant positions, capture probes, and stacking oligonucleotides. The $T_{m}$ values of these four capture probes were near $36^{\circ} \mathrm{C}$ (as listed in Table 1). The asymmetric PCR product from different plasmid was directly mixed with stacking oligonucleotides, HLASO2 and HLASO3, in hybridization buffer for pre-annealing with no further purification and allowed to hybridize to oligonucleotide microarrays at $40^{\circ} \mathrm{C}$ for $1 \mathrm{~h}$.

When the base mismatches were at the end of capture probes, the hybridization results in Figure 2 revealed differences in hybridization signal intensity between perfectly matched and mismatched duplexes, including G/T and

$\mathrm{A} / \mathrm{C}$ mismatches, which were the most difficult to discriminate by the classical allele-specific oligonucleotide (ASO) method on microarrays. HLA-A types 0206 and 2902 can be discriminated unambiguously on the same microarray. When the PCR products of HLA-A 2601 were hybridized with microarrays, the mismatch was in the middle of capture probe HLACP19, and the hybridization signal of HLACP19 was stronger than that of the capture probes with mismatches at the end. Therefore, the discrimination efficiency was decreased (Figure 2B). This provides another useful guide for designing capture probes in DNA sequence variation detection assisted by base stacking hybridization.

In conclusion, the goal of this study was to evaluate the effects of size-var-

HLA-A sequence 515-546

GCCCGGCGGA-T ${ }_{12}-\mathrm{NH}_{2}-5^{\prime}$ HLACP19

HLASO2: 3'-CCGAGAGGTGACGAGGCGGTGTACCCGGCGGA-T $12-\mathrm{NH}_{2}-5^{\prime}$, HLACP20 0206: 5'-GGCTCTCAACTGCTCCGCCACATGGGCCGCCT-3' 2601: 5'-GGCTCTCCACTGCTCCGCCTCATGGGCCGTCT-3' 2902: 5'-GGCTCTCAACTGCTCCGCCACACGGGCCGCCT-3'

HLA-A sequence 376-405

TGGGGTGCAG- $\mathrm{T}_{12}-\mathrm{NH}_{2}-5^{\prime}$ HLACP21

HLASO3: $3^{\prime}$ - CGCCTCCTTCGCGGGCAGGCCGGGGTGCAG- $\mathrm{T}_{12}-\mathrm{NH}_{2}-5^{\prime}$ HLACP22 0206: 5'- GCGGAGGAAGCGCCAGTCCGACCCCACGTC -3' 2601: 5'- GCGGAGGAAGCGCCCGTCCGGCCCCACGTC -3' 2902: 5'- GCGGAGGAAGCGCCCGTCCG $A$ CCCCACGTC -3'

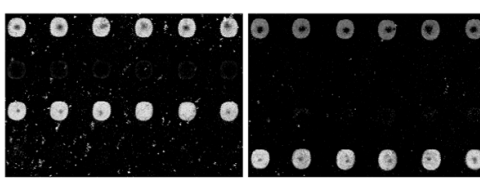

A

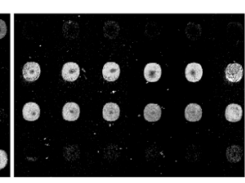

$\mathrm{C}$

Figure 2. HLA-A 0206, 2601, and 2902 typing on microarrays using stacking hybridization. The upper picture presents the sequences of HLA-A and capture probes. The italicized letters refer to the base variant sites. (A) Hybridization results of 0206; (B) hybridization results of 2601; and (C) hybridization results of 2902 . 
ied capture probes on mismatch discrimination using base stacking hybridization on microarrays to improve the efficiency of hybridization and sensitivity in detection. After evaluation, we considered the capture probes with a $\mathrm{T}_{\mathrm{m}}$ value of $36^{\circ} \mathrm{C}$ (11 nucleotides) and not $26^{\circ} \mathrm{C}$ (9 nucleotides) as reported previously (15) were the best capture probes for hybridization-based mismatch discrimination using base stacking hybridization on microarrays. In addition, by employing fluorescence asymmetric PCR to produce singlestranded target DNA for hybridization, we sufficiently simplified the overall process of mismatch discrimination using base stacking hybridization on microarrays. Furthermore, this improved method was applied for the typing of three HLA alleles, HLA-A 0206, 2601, and 2902 on microarrays. When the variant sites were at the end of capture probes, single-base mismatches could be discriminated distinctly, suggesting that the improved procedure was capable of simultaneously analyzing numerous sequence variations within one or more target DNA sequences on highthroughput oligonucleotide microarrays. This simple, fast, and reliable discrimination method has the potential to be employed in disease diagnosis and other practices. Current studies of these applications are under way, and the results will be reported in the future.

\section{ACKNOWLEDGMENTS}

This work was supported by the National Natural Science Foundation of China grant nos. 39889001 and 39825108, the National Key Basic Research Program of China no. G19990116, and the National Hi-Tech Program of China no. $2002 A A 2 Z 2011$ (J.C.). D.W. and H.G. contributed equally to this work.

\section{REFERENCES}

1.Vasiliskov, V.A., D.V. Prokopenko, and A.D. Mirzabekov. 2001. Parallel multiplex thermodynamic analysis of coaxial base stacking in DNA duplexes by oligodeoxyribonucleotide microchips. Nucleic Acids Res. 29:2303-2313.

2.Gellman, S.H., T.S. Haque, and L.F. Newcomb. 1996. New evidence that the hydrophobic effect and dispersion are not major driving forces for nucleotide base stacking. Biophys. J.
71:3523-3526.

3.Sponer, J., H.A. Gabb, J. Leszczynski, and P. Hobza. 1997. Base-base and deoxyribosebase stacking interactions in B-DNA and ZDNA: a quantum-chemical study. Biophys. J. 73:76-87.

4.Friedman, R.A. and B. Honig. 1995. A free energy analysis of nucleic acid base stacking in aqueous solution. Biophys. J. 69:1528-1535.

5.Norberg, J. and L. Nilsson. 1996. Influence of adjacent bases on the stacking-unstacking process of single-stranded oligonucleotides. Biopolymers 39:765-768.

6.Khrapko, K.R., Y. Lysov, A.A. Khorlin, I.B. Ivanov, G.M. Yershov, S.K. Vasilenko, V.L. Florentiev, and A.D. Mirzabekov. 1991. A method for DNA sequencing by hybridization with oligonucleotide matrix. DNA Seq. 1:375388 .

7.Yershov, G., V. Barsky, A. Belgovskiy, E. Kirillov, E. Kreindlin, I. Ivanov, S. Parinov, D. Guschin, et al. 1996. DNA analysis and diagnostics on oligonucleotide microchips. Proc. Natl. Acad. Sci. USA 93:4913-4918.

8.Parinov, S., V. Barsky, G. Yershov, E. Kirillov, E. Timofeev, A. Belgovskiy, and A. Mirzabekov. 1996. DNA sequencing by hybridization to microchip octa-and decanucleotides extended by stacked pentanucleotides. Nucleic Acids Res. 24:2998-3004.

9.Lysov, I.N., A.A. Chernyi, A.A. Balaev, F.N. Gnuchev, K.L. Beattie, and A.D. Mirzabekov. 1995. Use of continuous stacking hybridization in sequencing using modified oligonucleotide matrices. Mol. Biol. (Mosk) 29:104-113.

10.Lysov, I.P., A.A. Chernyi, A.A. Balaev, K.L. Beattie, V.L. Florent'ev, and A.D. Mirzabekov. 1993. Reconstruction of a sequenced sequence using results of stacked hybridization with an oligonucleotide matrix. Mol. Biol. (Mosk) 27:1126-1138.

11.Lysov, I.P., A.A. Chernyi, A.A. Balaev, F.N. Gnuchev, K.L. Beattie, and A.D. Mirzabekov. 1994. Effectiveness of sequencing using stacking hybridization on oligonucleotide matrices with varying length of immobilized oligonucleotides. Mol. Biol. (Mosk) 28:832839

12.Lysov, Y., A.A. Chernyi, A.A. Balaeff, K.L. Beattie, and A.D. Mirzabekov. 1994. DNA sequencing by hybridization to oligonucleotide matrix. Calculation of continuous stacking hybridization efficiency. J. Biomol. Struct. Dyn. 11:797-812.

13.Stomakhin, A.A., V.A. Vasiliskov, E. Timofeev, D. Schulga, R.J. Cotter, and A.D. Mirzabekov. 2000. DNA sequence analysis by hybridization with oligonucleotide microchips: MALDI mass spectrometry identification of 5mers contiguously stacked to microchip oligonucleotides. Nucleic Acids Res. 28:1193-1198

14.Maldonado-Rodriguez, R., M. EspinosaLara, A. Calixto-Suarez, W.G. Beattie, and K.L. Beattie. 1999. Hybridization of glasstethered oligonucleotide probes to target strands preannealed with labeled auxiliary oligonucleotides. Mol. Biotechnol. 11:1-12.

15.Maldonado-Rodriguez, R., M. EspinosaLara, P. Loyola-Abitia, W.G. Beattie, and K.L. Beattie. 1999. Mutation detection by stacking hybridization on genosensor arrays. Mol. Biotechnol. 11:13-25.

16.Little, S. 1997. ARMs analysis of point mutations, p. 45-52. In G.R. Taylor (Ed.), Laboratory Methods for the Detection of Mutations and Polymorphisms in DNA. CRC Press, Boca Raton, FL.

17.O'Meara, D., Z. Yun, A. Sonnerborg, and J. Lundeberg. 1998. Cooperative oligonucleotides mediating direct capture of hepatitis $\mathrm{C}$ virus RNA from serum. J. Clin. Microbiol. 36:2454-2459.

18.O'Meara, D., P. Nilsson, P.A. Nygren, M. Uhlen, and J. Lundeberg. 1998. Capture of single-stranded DNA assisted by oligonucleotide modules. Anal. Biochem. 255:195203.

19.Khan, M.A. 1989. A brief review of the HLA system. J. Pak. Med. Assoc. 39:187-191.

20.Terasaki, P. and S. Takemoto. 1999. Impact of HLA system on organ transplantation: a critical review before the end of the millennium. Adv. Nephrol. Necker Hosp. 29:163-175.

Received 27 January 2003; accepted 2 April 2003.

Address correspondence to Jing Cheng, Department of Biological Sciences and Biotechnology, Tsinghua University, Beijing 100084, The People's Republic of China. email: jcheng@tsinghua.edu.cn 